\title{
Coagulation parameters are associated with the prognosis of immunoglobulin a nephropathy: a retrospective study
}

Ming Xia ${ }^{1,2}$, Di Liu ${ }^{1,2}$, Liang Peng ${ }^{1,2}$, Yan Li ${ }^{1,2}$, Haiyang Liu ${ }^{1,2}$, Lingzhi Wu ${ }^{1,2}$, Guochun Chen ${ }^{1,2}$, Yu Liu ${ }^{1,2}$ and Hong Liu ${ }^{1,2^{*}}$ (iD

\begin{abstract}
Background: Interstitial fibrosis/tubular atrophy (T) score is a known determinant of the progression of immunoglobulin A nephropathy (IgAN). Strong evidence indicates that the components of the coagulation system closely linked with fibrotic events have been highlighted in the kidney. However, whether the coagulation system can affect the renal outcome of IgAN remains unclear. Herein, we investigated the association of coagulation parameters and pathological phenotype of IgAN and their combined effects on the deterioration of renal function.

Methods: This retrospective study included N=291 patients with biopsy-proven IgAN from May 2009 to April 2013 in the Second Xiangya Hospital. Clinical data, pathological features were collected, and the associations of coagulation parameters at biopsy, T score, and renal outcome were evaluated. T score indicated the degree of tubular atrophy or interstitial fibrosis. The renal outcome was defined as an end-stage renal disease (ESRD) or an irreversible 50\% estimated glomerular filtration rate (eGFR) reduction.

Results: Shorter prothrombin time (PT) and the activated partial thromboplastin time (APTT) were significantly associated with T (both $p<0.001)$. PT $(<11.15 \mathrm{~s})$ or APTT $(<29.65 \mathrm{~s})$ had worse cumulative survival rate $(p=0.008, p=0.027$ respectively) and were significantly but not independently associated with a higher risk of renal outcome ( $p=$ $0.012, p=0.032$ respectively). In the combined analyses of PT, APTT, and T lesions, the odd ratios for the outcome were significantly higher in the presence of T with PT (<11.15 s) or APTT $(<29.65$ s).

Conclusion: Shorter PT and APTT are associated with an increased incidence of the T lesion and are additional factors that portend a poorer prognosis in IgAN. Monitoring coagulation function might be important when assessing the risk of progression. Additional studies exploring the molecular mechanism between coagulation and IgAN pathology are needed.
\end{abstract}

Keywords: Coagulation, Fibrosis, IgA nephropathy, Prognosis

\footnotetext{
* Correspondence: liuhong618@csu.edu.cn

'Department of Nephrology, The Second Xiangya Hospital, Central South University, 139 Renmin Road, Changsha 410011, Hunan, China

${ }^{2}$ Hunan Key Laboratory of Kidney Disease and Blood Purification, 139 Renmin Road, Changsha 410011, Hunan, China
}

(c) The Author(s). 2020 Open Access This article is licensed under a Creative Commons Attribution 4.0 International License, which permits use, sharing, adaptation, distribution and reproduction in any medium or format, as long as you give appropriate credit to the original author(s) and the source, provide a link to the Creative Commons licence, and indicate if changes were made. The images or other third party material in this article are included in the article's Creative Commons licence, unless indicated otherwise in a credit line to the material. If material is not included in the article's Creative Commons licence and your intended use is not permitted by statutory regulation or exceeds the permitted use, you will need to obtain permission directly from the copyright holder. To view a copy of this licence, visit http://creativecommons.org/licenses/by/4.0/. The Creative Commons Public Domain Dedication waiver (http://creativecommons.org/publicdomain/zero/1.0/) applies to the data made available in this article, unless otherwise stated in a credit line to the data. 


\section{Background}

Immunoglobulin A nephropathy (IgAN) is one of the most prevalent primary glomerulonephritis worldwide, especially, accounting for $\sim 40 \%$ in Asia [1], and $~ 50 \%$ in China [2]. It is the main cause of end-stage renal failure (ESRD), approximately $30-40 \%$ of IgAN patients develop ESRD, which brings a heavy burden to individuals and society [3]. Therefore, it is of great scientific and practical significance to identify the risk factors associated with the progression of IgAN. The oxford classification MEST-C score is well regarded prognostic indicators for IgAN, containing the presence of mesangial hypercellularity (M), endocapillary hypercellularity (E), segmental glomerulosclerosis (S), tubular atrophy/interstitial fibrosis $(\mathrm{T})$, crescent $(\mathrm{C})$ based on renal histopathology $[4,5]$. Of them, the $\mathrm{T}$ score is the most valuable histological parameter, confirmed by a large number of original studies [6-8]. T lesion is not merely a histomorphological feature of IgAN but is rather a final common pathway for most progressive kidney diseases and leads to advanced chronic kidney disease (CKD) [9, 10]. However, factors associated with tubulointerstitial damage of IgAN have not been elucidated.

Given the potential role of the coagulation system in the development of renal fibrosis, the regeneration and proliferation of tubular or mesangial cells [11-13], it is interesting and clinically useful to explore whether coagulation parameters are linked to the pathological Oxford classification of IgAN. In this study, we sought to investigate two key concepts. First, are coagulation parameters at the time of renal biopsy associated with $\mathrm{T}$ score? Second, how much do the coagulation parameters affect the renal outcome? To investigate this, we identified a cohort of patients with biopsy-proven IgAN and collected their clinical and laboratory parameters.

\section{Methods}

\section{Subjects}

Two hundred and ninety-one patients with a biopsybased diagnosis of primary IgAN between May 2009 and April 2013 were recruited at the Second Xiangya Hospital of Central South University. A flow diagram of the selection of the participants is presented in Fig. 1. The inclusion criteria were the initial estimated glomerular filtration rate $(e G F R) \geq 15 \mathrm{~mL} / \mathrm{min} / 1.73 \mathrm{~m}^{2}$ at the time of renal biopsy and without receiving any corticosteroids or immunosuppressants before the renal biopsy. The exclusion criteria included (1) a biopsy specimen containing less than eight glomeruli, (2) patients with secondary IgAN, such as Henoch-Schonlein purpura, lupus nephritis, hepatic disease or (3) with ESRD on admission, (4) patients with missing clinical data at the time of renal biopsy and follow-up data or (5) complicated with other diseases and taking medication that may affect coagulation function. The outcome in this study was the occurrence of ESRD or a reduction of eGFR 50\% from the baseline. The study was performed with the informed consent of all patients, and the procedure was approved by the Ethics Committee of the Second Xiangya Hospital, Central South University, and is in accordance with the principles of the Declaration of Helsinki.

\section{Clinical and pathological measures}

The following parameters were collected at baseline for each patient: age, gender, systolic blood pressure and mean arterial pressure (SBP and MAP respectively) serum creatinine $(\mathrm{Scr})$, eGFR (modified Modification of Diet in Renal Disease equation) [14], 24 h proteinuria, coagulation parameters including activated partial thromboplastin time (APTT), prothrombin time (PT), thrombin time (TT), fibrinogen degradation products

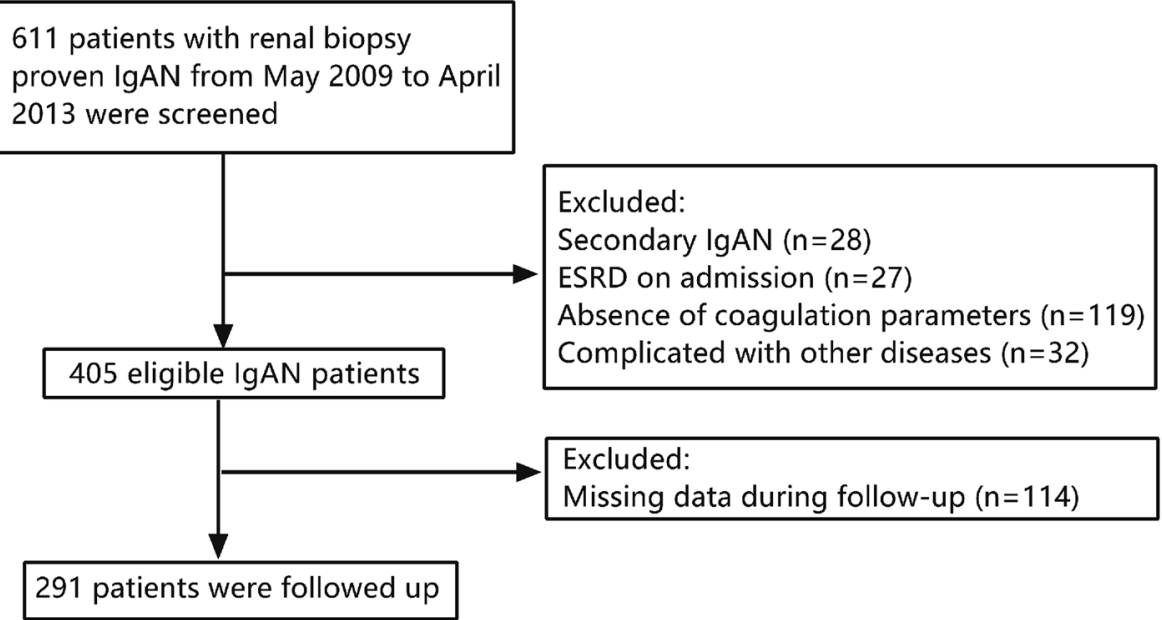

Fig. 1 A flow diagram of the strategy used to identify IgAN patients 
(FDP), fibrinogen (FIB), D-dimer (D-D), antithrombin III antibodies (AT-III).

All Renal biopsies were scored according to the new Oxford classification of the IgAN scoring system (MEST-C scoring system) proposed by the IgA Nephropathy Classification Working Group and two pathological doctors confirmed the pathological results [4]. The histologic classification was performed as follows: M0 or M1 indicated the mesangial score $\leq 0.5$ or $>0.5$, respectively; E0 and E1 indicated the absence or presence of endocapillary hypercellularity, respectively; S0 or S1 indicated the absence or presence of segmental glomerulosclerosis, respectively; T0, $\mathrm{T} 1$, and $\mathrm{T} 2$ indicated the degree of tubular atrophy or interstitial fibrosis $(<25 \%, 25-50 \%$, and $>50 \%$, respectively). C0, $\mathrm{C} 1$, and $\mathrm{C} 2$ indicated no crescent in glomeruli, crescent in $<25 \%$ of glomeruli, and crescent in $>25 \%$ of glomeruli, respectively.

\section{Statistical analysis}

The numerical variables are expressed as the means with standard deviations (SD) or medians with interquartile ranges (IQR), and the categorical variables are expressed as count (\%). The Chi-square test was adopted for analyzes of categorical variables and continuous variables were compared by using Student's t-test or KruskalWallis tests. The coagulation parameters cut-off values were determined by the receiver operating characteristic (ROC) curve. The Kaplan-Meier survival analysis was performed to compare the survival rate by using the logrank test. Cox proportional hazards regression was used to perform univariate and multivariate analyses to identify prognostic factors and estimate the hazard ratios (HRs) and 95\% confidence intervals (95\% CIs). Binary logistic regression was used to explore the effect on the progression of IgAN in different groups. $P$-value $<0.05$ was considered statistically significant. The analyses were performed using SPSS statistics 22.0 software (SPSS Inc., Chicago, IL, USA) and Graphed Prism 6 (GraphPad Software Inc., San Diego, CA, USA).

\section{Results}

\section{Baseline characteristics of participants}

A total of 611 biopsy-proven IgAN patients between May 2009 and April 2013 were initially screened in the study, and 291 patients were enrolled in this retrospective cohort study according to the inclusion and exclusion criteria (shown in Fig. 1). The median follow-up time was 41.2 months. Patients were divided into two groups based on the T score, and the baseline characteristics and histopathological features at the time of renal biopsy were shown in Table 1 . Of all 291 patients, 131 (45.02\%) were males, and the mean age was $32.50 \pm$ 11.94 years. The mean SBP, MAP, Scr, eGFR and $24 \mathrm{~h}$ urine protein were $127.18 \pm 11.95 \mathrm{mmHg}, \quad 96.3 \pm 12.75 \mathrm{mmHg}$, $92.73 \pm 47.14 \mu \mathrm{mol} / \mathrm{L}, 90.87 \pm 69.14 \mathrm{~mL} / \mathrm{min} / 1.73 \mathrm{~m}^{2}, 1.71 \pm$ $2.51 \mathrm{~g} /$ day, respectively. Regarding MEST-C oxford scores in all patients, $6.53 \%$ were $\mathrm{M} 1,11 \%$ were $\mathrm{E} 1,66.67 \%$ were $\mathrm{S} 1$, $40.2 \% \mathrm{~T} \geq 1$, and $21.99 \% \mathrm{C} \geq 1$. Compared to the absence of $\mathrm{T}$ lesion group, IgAN patients with $\mathrm{T}$ had significantly higher levels of SBP, MAP $(129.17 \pm 16.21 \mathrm{mmHg}$ vs $125.84 \pm 16.05 \mathrm{mmHg}$, and $98.40 \pm 12.97 \mathrm{mmHg}$ vs $94.90 \pm$ $12.43 \mathrm{mmHg}), \quad$ Scr $\quad(115.35 \pm 59.31 \mu \mathrm{mol} / \mathrm{L} \quad$ vs $77.52 \pm$ $28.05 \mu \mathrm{mol} / \mathrm{L})$, and lower eGFR $(80.1 \pm 102.43 \mathrm{~mL} / \mathrm{min} / 1.73$ $\mathrm{m}^{2}$ vs $98.11 \pm 29.11 \mathrm{~mL} / \mathrm{min} / 1.73 \mathrm{~m}^{2}$ ). There was also a tendency of difference in $24 \mathrm{~h}$ proteinuria between two groups $1.62 \pm 2.20 \mathrm{~g} / 24 \mathrm{~h}$ vs $1.77 \pm 2.71 \mathrm{~g} / 24 \mathrm{~h}$ ). For renal biopsy, 6 (5.13\%) patients had M1, 15 (12.82\%) had E1, 100 (85.47\%) had S1, 30 (25.64\%) had C1 or C2 in presence of $\mathrm{T}$ group. 13 (7.47\%) of absence $\mathrm{T}$ patients had M1, 17 (9.77\%) had E1, 94 (54.02\%) had S1, 34 (19.54\%) had C1 or C2, and percentage of S1 was significantly lower compared to the

Table 1 Clinical characteristics and histopathological features of study participants with and without tubular atrophy/interstitial fibrosis $(T)$

\begin{tabular}{|c|c|c|c|c|}
\hline Characteristics & $\begin{array}{l}\text { All participants } \\
(n=291)\end{array}$ & $\begin{array}{l}\text { IgAN without T } \\
(\mathrm{n}=174)\end{array}$ & $\begin{array}{l}\text { IgAN with T } \\
(n=117)\end{array}$ & $p$ value \\
\hline MAP $(\mathrm{mmHg})$ & $96.30(12.75)$ & $94.90(12.43)$ & $98.40(12.97)$ & 0.023 \\
\hline $\operatorname{Scr}(\mu \mathrm{mol} / \mathrm{L})$ & $92.73(47.14)$ & $77.52(28.05)$ & $115.35(59.31)$ & $<0.001$ \\
\hline eGFR $\left(\mathrm{mL} / \mathrm{min} / 1.73 \mathrm{~m}^{2}\right)$ & $90.87(69.14)$ & $98.11(29.11)$ & $80.10(102.43)$ & $<0.001$ \\
\hline Proteinuria (g/24 h) & $1.71(2.51)$ & $1.77(2.71)$ & $1.62(2.20)$ & 0.056 \\
\hline \multicolumn{5}{|l|}{ Oxford classification } \\
\hline M0/M1 (\% of M1) & $272 / 19(6.53)$ & $161 / 13(7.47)$ & $111 / 6(5.13)$ & 0.428 \\
\hline E0/E1 (\% of E1) & 259/32 (11.00) & 157/17 (9.77) & 102/15 (12.82) & 0.415 \\
\hline so/S1 (\% of S1) & 97/194 (66.67) & $80 / 94(54.02)$ & 17/100 (85.47) & $<0.001$ \\
\hline $\mathrm{T} 0 / \mathrm{T} 1 / \mathrm{T} 2$ (\% of [T1 + T2]) & 174/93/24 (40.2) & - & - & - \\
\hline $\mathrm{C} 0 / \mathrm{C} 1 / \mathrm{C} 2(\%$ of $[\mathrm{C} 1+\mathrm{C} 2])$ & 227/56/8 (21.99) & 140/30/4 (19.54) & $87 / 26 / 4(25.64)$ & 0.46 \\
\hline
\end{tabular}

The data were presented as mean (SD) or count (percentage). Abbreviations: SBP systolic blood pressure, MAP mean arterial pressure, Scr serum creatinine, eGFR estimated glomerular filtration rate. $P$ values in bold denote significance at the 0.05 level 
presence of $\mathrm{T}$ group. There were no significant differences in age, gender, $\mathrm{M}, \mathrm{E}, \mathrm{C}$ scores between the two groups.

\section{Relationships between coagulation parameters and $\mathrm{T}$ score}

The association of coagulation parameters, including PT, APTT, TT, FDP, FIB, D-D, AT-III, and T lesion were investigated. For all IgAN patients, 21 patients had shorter PT seconds and 16 patients had longer seconds than the reference value. For APTT, 41 of IgAN patients were shorter and 46 patients were longer than the reference. There were 22 patients with both abnormal PT and APTT levels. As is shown in Table 2, PT and APTT seconds of patients with $\mathrm{T}$ was significantly shorter (both $p<0.001)$ than that of the without $\mathrm{T}$ group. There were no statistical differences in TT, FDP, FIB, D-D, and ATIII levels. We also explored the relationship between PT, APTT, and other oxford classification of IgAN, but no significant differences were found. In addition, we would like to know whether PT and APTT are related to the degree of $\mathrm{T}$ rating. In the comparison of the T0 group, PT and APTT were significantly shorter in T1 $(p=0.003$ and $p=0.012$, respectively) and T2 group $(p<0.001$ and $\mathrm{p}=0.003$, respectively). Moreover, $\mathrm{PT}$ also showed a statistical difference between T1 and T2 group ( $p=$ 0.036) (shown in Fig. 2).

\section{Relationships between PT, APTT, and renal outcome}

Of all the participants, a 50\% decline in eGFR was observed in $4.8 \%(n=14)$ of the patients, and $2.4 \%(n=7)$ of the patients developed ESRD during the follow-up period. In order to investigate the association of PT, APTT, and renal progression, ROC curves were firstly constructed (shown in Fig. 3). The areas under the ROC curves (AUCs) for PT and APTT were 0.67 (95\%CI $0.54-0.79$ ) and 0.7 (95\%CI 0.59-0.79). Based on PT and APTT cut-off value, patients were divided into PT $<$ $11.15 \mathrm{~s}$ and $>11.15 \mathrm{~s}$ groups, or APTT $<29.65 \mathrm{~s}$ and $>$ $29.65 \mathrm{~s}$ groups respectively. Kaplan-Meier survival analysis was performed to examine renal survival between groups (shown in Fig. 4). The cumulative survival rate was significantly higher in patients with longer PT (> $11.15 \mathrm{~s}$ ) or APTT $(>29.65 \mathrm{~s})$ than the lower groups $(p=$ $0.008, p=0.027$ respectively).

Cox proportional hazards models were constructed to determine the prognostic value of PT, APTT, and clinical/histopathological parameters in IgAN patients (Table 3). Univariate analysis showed higher values of Scr (HR 1.22, 95\%CI 1.16-1.28, per 10umol/L increase), SBP (HR 1.34, 95\% CI 1.08-1.67, per $10 \mathrm{mmHg}$ increase), $24 \mathrm{~h}$ proteinuria (HR $1.13,95 \% \mathrm{CI} 1.00-1.28$, per $1 \mathrm{~g}$ increase) and lower values of eGFR (HR $0.52,95 \% \mathrm{CI}$ 0.41-0.66, per $1 \mathrm{~mL} / \mathrm{min} / 1.73 \mathrm{~m} 2$ increase), PT (HR $3.27,95 \%$ CI $1.30-8.24$ versus $>11.15 \mathrm{~s}$ group), APTT (HR 2.59, 95\%CI $1.08-6.21$ versus $>29.65 \mathrm{~s}$ group) at baseline were significantly associated with a higher risk of renal outcome. The biopsy score S1 (HR 3.68, 95\% CI 1.08-12.59) and $\mathrm{T} \geq 1$ (HR 13.16, 95\%CI 3.06-56.67) also significantly correlated to poor prognosis. However, after adjusting for univariate risk factors, the multivariate analysis showed no significant value in PT or APTT (data not shown).

Thus, we assessed the additive effect of PT or APTT and $\mathrm{T}$ lesion on the renal outcome (shown in Fig. 5). Patients without $\mathrm{T}$ lesion and with $\mathrm{PT}>11.15 \mathrm{~s}$ or APTT $>$ $29.65 \mathrm{~s}$ were set as the reference groups. The odd ratios (ORs) were 3 (95\%CI: $0.18-49.00 ; P=0.44)$ for patients without $\mathrm{T}$ and $\mathrm{PT}<11.15 \mathrm{~s}, 13.35$ (95\%CI: $1.57-113.37$; $P=0.018)$ for patients with $\mathrm{T}$ and $\mathrm{PT}>11.15 \mathrm{~s}$, and 41.93 (95\%CI: 5.32-330.48; $P<0.001$ ) for patients with $\mathrm{T}$ and $\mathrm{PT}<11.15 \mathrm{~s}$. This association was consistent with the combination of APTT and T lesion. The corresponding ORs were 5.88 (95\%CI: $0.36-97.09 ; P=0.22$ ), 22.15 (95\%CI: $2.81-174.89, \quad P=0.003)$, and 47.04 (95\%CI: 5.64-392.55; P<0.001).

\section{Discussion}

Considering that $\mathrm{T}$ score is a well-recognized independent predictor of IgAN, it is worth exploring the possible risk factors associated with T. Studies have reported that

Table 2 Comparison of coagulation parameters in IgAN patients without or with tubular atrophy/interstitial fibrosis (T)

\begin{tabular}{lllll}
\hline Factors & $\begin{array}{l}\text { All participants } \\
(\mathbf{n}=\mathbf{2 9 1})\end{array}$ & $\begin{array}{l}\text { IgAN without T } \\
(\mathbf{n = 1 7 4 )}\end{array}$ & $\begin{array}{l}\text { IgAN with T } \\
(\mathbf{n}=\mathbf{1 1 7})\end{array}$ & $\mathbf{p}$ value \\
\hline PT $(\mathrm{s})$ & $11.80(10.80-12.70)$ & $12.00(11.10-12.80)$ & $11.40(10.45-12.30)$ & $<0.001$ \\
APTT $(\mathrm{s})$ & $36.40(31.00-41.80)$ & $38.60(32.38-43.83)$ & $35.20(29.15-39.25)$ & $<0.001$ \\
TT $(\mathrm{s})$ & $16.80(15.50-18.60)$ & $16.75(15.40-18.20)$ & $17.10(15.55-19.40)$ & 0.075 \\
FDP $(\mu \mathrm{g} / \mathrm{ml})$ & $1.50(1.00-2.30)$ & $1.50(1.00-2.50)$ & $1.40(1.00-2.00)$ & 0.3 \\
FIB $(\mathrm{g} / \mathrm{l})$ & $3.07(2.54-3.87)$ & $3.03(2.54-3.92)$ & $3.10(2.53-3.84)$ & 0.964 \\
D-D $(\mu \mathrm{g} / \mathrm{ml})$ & $0.44(0.25-0.81)$ & $0.40(0.23-0.83)$ & $0.50(0.30-0.77)$ & 0.188 \\
AT-III $(\%)$ & $98.90(87.00-111.00)$ & $98.20(85.45-109.28)$ & $100.10(88.65-113.50)$ & 0.089 \\
\hline
\end{tabular}

The data were presented as median (IQR). Abbreviations: $P T$ prothrombin time, APTT activated partial thromboplastin time, $\Pi T$ thrombin time, $F D P$ fibrinogen degradation products, FIB fibrinogen, D-D D-dimer, AT-III antithrombin III antibodies. P values in bold denote significance at the 0.05 level 

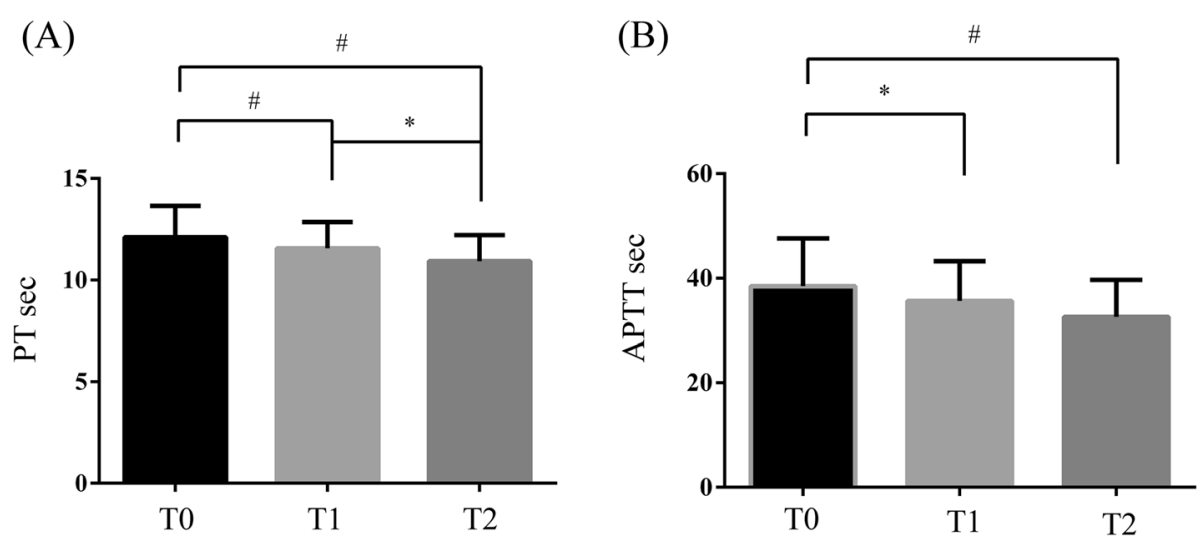

Fig. 2 Comparison of (a) PT and (b) APTT among T0, T1 and T2 IgAN patients. T0 group ( $n=174)$, T1 group ( $n=93)$ and T2 group ( $n=24)$. The data were presented as mean \pm SD. ${ }^{*} p<0.05$, \# $p<0.01$

tubulointerstitial injury of IgAN was associated with trefoil factor 3 mRNA [15], p38 MAPK activity [16], BMI [17], serum matrix metalloproteinase-7 level [18]. In this study, we demonstrated the significance of coagulation parameters PT and APTT in the tubulointerstitial injury of IgAN.

Strong evidence indicates that the components of coagulation factors may cause ischemia and localized blood flow disruption through the formation of microthrombus, and drive pro-fibrotic events [19]. Moreover, anticlotting drugs were reported to reduce fibronectin accumulation and improve renal fibrosis $[20,21]$. Actually, the coagulation system is a complex balance of coagulant and anticoagulant components that functions in unique homeostasis by interacting with activated endothelial cells, coagulation factors, etc. [22]. In the kidney, when microvascular endothelial cells are abnormally damaged, the activation of platelets and the release of plasma coagulation factors can activate intra-glomerular coagulation [23]. PT, APTT, TT, FDP, FIB, D-D, and AT-III measurements are routine coagulation tests used to assess coagulation systems prior to renal biopsy. The APTT and the PT reflect the function of endogenous and exogenous pathways of the coagulation cascade [24], and its duration could be affected by the upstream Xa factor common to coagulation activation pathways. Reports suggested that factor Xa could cooperate with $\mathrm{V}$ to exert procoagulant activity in active IgAN, leading to intra-mesangial coagulation $[25,26]$. Prothrombin/ thrombin has been reported to be elevated in acute kidney injury (AKI), nephrotic syndrome, CKD and other renal diseases, inhibition could attenuate tubular atrophy or proliferative responses in renal [27]. However, no study has shed light on the relationship between coagulation parameters and clinical or pathological data in IgAN. In our study, APTT and PT were found significantly shorter (both $p<0.001$ ) in IgAN patients with tubular atrophy/interstitial fibrosis $(\mathrm{T})$ when compared with the absence of $\mathrm{T}$ group, suggesting the activation of endogenous and exogenous pathways of the coagulation cascade. This also suggests that renal fibrosis may be accompanied by a hypercoagulable state, which is consistent with the coagulation situation found in patients with pulmonary fibrosis or cirrhosis, and anticoagulation as a therapeutic option is promising [28-30].

Glomerular FIB activation is a secondary event after vascular injury in glomerular clusters. Urinary fibrinogen was shown to correlate with interstitial fibrosis and was

\section{(A)}

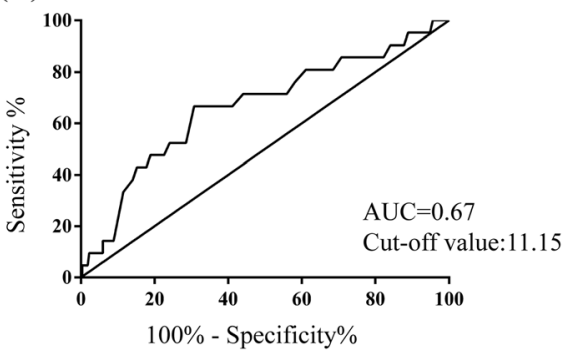

(B)

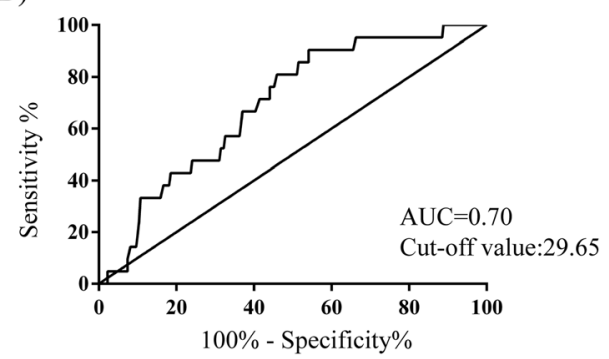

Fig. 3 ROC curves of (a) PT, (b) APTT for the identification of poor renal outcome. The best cut-off value for PT and APTT are labeled on the plot 
(A)

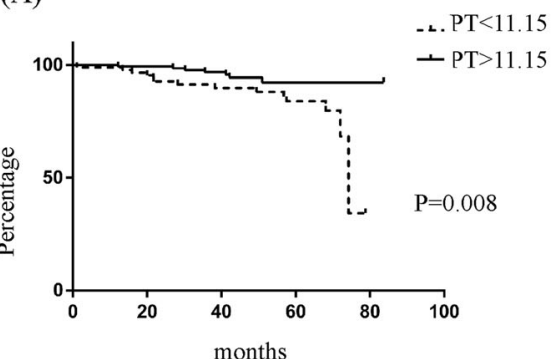

(B)

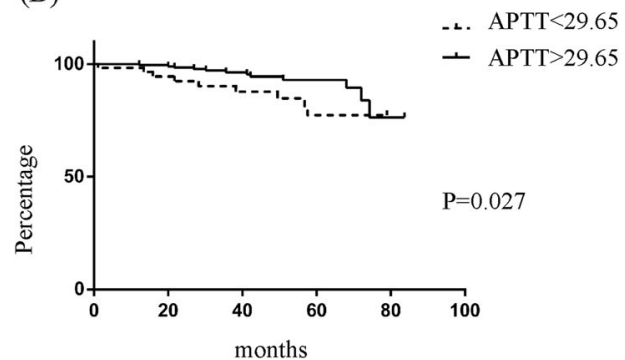

Fig. 4 Kaplan-Meier curves of ESRD/50\% drop in eGFR from baseline according to (a) PT, (b) APTT levels category

an independent risk factor for the progression of CKD to ESRD [31]. TT reflects the ability of subjects to convert plasma FIB to fibrin [32]. In IgAN, soluble fibrin deposition was reported to be found in kidney tissue specimens in its early stage [33]. In our results, there was no significant difference in FIB and TT between IgAN with $\mathrm{T}$ and without groups. AT-III, D-D, FDP are indicators of the activity status of the fibrinolytic system. AT-III is an important anticoagulant in the body and participates in the dynamic balance of the coagulation and fibrinolytic systems, its administration was shown beneficial for interstitial fibrosis in AKI model [34]. In IgAN patients, we found no difference in the AT-III level between the IgAN with $\mathrm{T}$ group and without $\mathrm{T}$ group, suggesting that the anticoagulant system was not obviously altered when the presence of fibrosis. D-D and FDP are the degradation product of fibrin, both of which reflect the fibrinolytic function [35]. No difference was observed between the two groups, indicating that interstitial fibrosis/tubular atrophy was not accompanied by a hyper/lower activity of fibrinolysis. Apart from coagulation parameters, SBP, MAP, Scr, eGFR, and S score were also associated with the degree of renal interstitial fibrosis in IgAN from our results.

In our previous study, serum D-D was found to serve as a potential predictor for thrombotic events in patients with kidney disease [36]. For IgAN, Scr, eGFR, proteinuria, and pathology characteristics have been identified as baseline predictors of progression [8, 37-39], which is consistent with our findings. As coagulation function showed an association with the $\mathrm{T}$ score of pathology as described, and could be well monitored in a clinical laboratory, it is of clinical significance to explore the relationship between coagulation function and the prognosis

Table 3 Association between baseline characteristics of patients and the incidence of renal outcome

\begin{tabular}{|c|c|c|c|c|}
\hline Baseline variables & HR & 95\% Lower & 95\% Upper & $p$ value \\
\hline \multicolumn{5}{|l|}{ Demographics } \\
\hline Age, per 10 year & 0.81 & 0.55 & 1.20 & 0.29 \\
\hline Gender (male versus female) & 0.98 & 0.41 & 2.33 & 0.964 \\
\hline \multicolumn{5}{|l|}{ Laboratory measures at baseline } \\
\hline Scr, per 10 umol/L & 1.22 & 1.16 & 1.28 & $<0.001$ \\
\hline eGFR, per $10 \mathrm{~mL} / \mathrm{min} / 1.73 \mathrm{~m}^{2}$ & 0.52 & 0.41 & 0.66 & $<0.001$ \\
\hline SBP, per $10 \mathrm{mmHg}$ & 1.34 & 1.08 & 1.67 & 0.007 \\
\hline $24 \mathrm{~h}$ proteinuria, per $1 \mathrm{~g}$ & 1.13 & 1.00 & 1.28 & 0.05 \\
\hline PT $(<11.15 \mathrm{~s}$ versus $>11.15 \mathrm{~s})$ & 3.27 & 1.30 & 8.24 & 0.012 \\
\hline APTT $(<29.65 \mathrm{~s}$ versus $>29.65 \mathrm{~s})$ & 2.59 & 1.08 & 6.21 & 0.032 \\
\hline \multicolumn{5}{|l|}{ Histology at biopsy } \\
\hline M (1 versus 0$)$ & 0.05 & 0.00 & 223.16 & 0.476 \\
\hline E (1 versus 0$)$ & 0.79 & 0.18 & 3.41 & 0.752 \\
\hline $\mathrm{S}(1$ versus 0$)$ & 3.68 & 1.08 & 12.59 & 0.038 \\
\hline $\mathrm{T}(\geq 1$ versus 0$)$ & 13.16 & 3.06 & 56.67 & 0.001 \\
\hline$C(\geq 1$ versus 0$)$ & 1.58 & 0.61 & 4.10 & 0.344 \\
\hline
\end{tabular}

Abbreviations: SBP systolic blood pressure, Scr serum creatinine, eGFR estimated glomerular filtration rate. P values in bold denote significance at the 0.05 level 
(A)

* $\mathrm{p}<0.05$ vs $\mathrm{PT}>11.15 \mathrm{~s}$ without $\mathrm{T}$

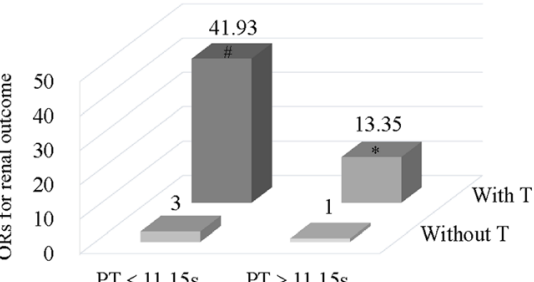

(B) $\#$ p $<0.05$ vs APTT $>29.65$ s without $T$

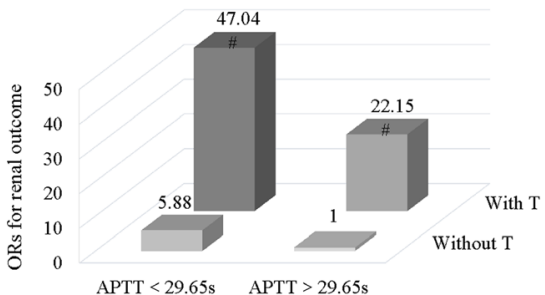

Fig. 5 The analysis of the additive effect of (a) PT, (b) APTT, and T score on the renal outcome. The renal outcome was defined as an ESRD or an irreversible 50\% eGFR reduction. X-axis stood for subgroups of PT or APTT. Z-axis stood for with/without T, and the Y-axis stood for the OR value. Patients without T and PT $>11.15 \mathrm{~s}$ or APTT $>29.65 \mathrm{~s}$ were set as the reference, respectively. ${ }^{*} p<0.05, \# p<0.01$

of IgAN. The shorter PT $(<11.15 \mathrm{~s})$ and APTT $(<29.65$ s) showed a significantly worse prognosis compared to the longer group according to the Kaplan-Meier survival curve. Although univariate cox analysis of PT and APTT was significant, the multivariate analysis adjusted for univariate risk factors was not. This indicates that PT and APTT have limited ability as independent predictors but maybe secondary factors that increase the risk of poor prognosis in IgAN. The analysis of the combination of a shorter PT or APTT with T lesion could adversely affect the renal outcome more in IgAN also supports that shorter PT and APTT indirectly accelerated the progression.

It is well known that nephrotic-range proteinuria is related to acquired complex hypercoagulability involving the acceleration of the entire thrombotic process (activated intrinsic pathway, fibrinogen, platelet function and fibrin-platelet interaction) [40, 41]. Hypercoagulability was found highly correlated with the severity of nephropathy and likely secondary to nephrosis [42]. Although no clear molecular therapeutic targets have been identified, most studies have shown that the main pathological changes involve antithrombin, fibrinogen, and factors $\mathrm{V}$ and VIII [43]. Also, in most CKD patients, coagulation disorder is an important non-immune factor for the occurrence and disease development [44]. Correcting coagulation imbalance is of great significance for early CKD stage treatment, and direct oral anticoagulants use in patients with ESRD and advanced CKD is increasing [45]. For IgAN, there is insufficient evidence to determine the causal relationship between coagulation and disease progression. Although early studies of anticoagulation therapy showed that warfarin combined with dipyridamole was not effective in IgAN treatment [46], the combination therapy including prednisolone, mizoribine with warfarin and dipyridamole were found better for severe childhood IgAN than the combination treatment including prednisolone and mizoribine without warfarin or dipyridamole from the point of proteinuria remission [47]. This suggests that anticoagulant may be promising as an adjunctive drug in IgAN, and determine the relationship between hypercoagulability and markers of disease severity may help to identify clinically meaningful towards when and how anticoagulants warranted.

To the best of our knowledge, this is the first paper that focuses on the relationship between coagulation function, T score, and IgAN prognosis. Possible mechanisms to explain the association between shorter PT or APTT and interstitial fibrosis/tubular atrophy are as follows. (1) With the progression of IgAN, renal impairment is aggravated, renal units are damaged accompanied by fibrosis, resulting in loss of normal excretory function and reduced clearance of procoagulant substances. (2) Endothelial injury is a common manifestation in IgAN $[48,49]$, the shortening of PT or APTT may be related to the initiation of coagulation by vascular endothelial injury. (3) Moreover, extensive researches have found that coagulation is related to inflammatory response, and IgAN is also an inflammatory disease. IgAN patients are commonly associated with alteration of various inflammatory cytokines [50], which may be related to the activation of procoagulant factors and renal fibrosis [51, 52]. In addition, there are some limitations to our study. First, the data were collected at a single center, which may limit the generalisability of the results. Second, although those on treatment with agents affecting coagulation parameters were excluded, we did not consider treatment with angiotensin-converting enzyme inhibitors/angiotensin receptor blockers or antihypertension therapy and how this may affect the results and vascular physiology.

In conclusion, our study reveals a novel aspect of tubular atrophy/interstitial fibrosis (T), linking coagulation function to clinical and pathological of IgAN. Shorter PT and APTT are associated with the presence of $\mathrm{T}$ and may increase the risk of poor prognosis in IgAN. The molecular mechanism between coagulation and IgAN pathology needs to be further explored. 


\section{Abbreviations}

IgAN: Immunoglobulin A nephropathy; ESRD: End-stage renal disease; CKD: Chronic kidney disease; M: Mesangial hypercellularity; E: Endocapillary hypercellularity; S: Segmental glomerulosclerosis; T: Interstitial fibrosis/tubular atrophy; C: Crescent; eGFR: Estimated glomerular filtration rate; SBP: Systolic blood pressure; MAP: Mean arterial pressure; Scr: Serum creatinine; APTT: Activated partial thromboplastin time; PT: Prothrombin time; ПT: Thrombin time; FDP: Fibrinogen degradation products; FIB: Fibrinogen; D-D: D-dimer; AT-III: Antithrombin III antibodies; SD: Standard deviations; IQR: Interquartile ranges; ROC: Receiver operating characteristic; HR: Hazard ratios; $\mathrm{Cl}$ : Confidence intervals; AUC: Areas under the ROC curve; OR: Odd ratios

\section{Acknowledgments}

Not applicable.

\section{Authors' contributions}

$M X, D L$, and LP contributed to study design and drafting the manuscript; YL1, HYL, and LZW contributed to data collection and statistical analysis/ interpretation; GCC and YL2 contributed to review and editing; HL was responsible for the supervision and final approval of the version to be published. We sincerely appreciate the time and effort of all who contributed to this study. The authors read and approved the final manuscript.

\section{Funding}

This work was supported by research grants (81770714) and (81570622) from the National Natural Science Foundation of China. These funds were used to maintain the database operation, update the statistical software, and pay patients follow-up fee.

\section{Availability of data and materials}

The datasets used and/or analyzed during the current study are available from the corresponding author on reasonable request.

\section{Ethics approval and consent to participate}

The research was in compliance with the Declaration of Helsinki and was approved by the ethical committee of the Second Xiangya Hospital. All participants were informed about the purpose of this study and signed written informed consent.

\section{Consent for publication}

Not applicable.

\section{Competing interests}

The authors declare that they have no competing interests.

\section{Received: 7 June 2020 Accepted: 18 October 2020}

Published online: 27 October 2020

\section{References}

1. Chen T, Li X, Li Y, Xia E, Qin Y, Liang S, et al. Prediction and risk stratification of kidney outcomes in IgA nephropathy. Am J Kidney Dis. 2019;74(3):300-9.

2. O'Shaughnessy MM, Hogan SL, Thompson BD, Coppo R, Fogo AB, Jennette JC. Glomerular disease frequencies by race, sex and region: results from the international kidney biopsy survey. Nephrol Dial Transplant. 2018;33(4):661-9.

3. Lai KN, Tang SC, Schena FP, Novak J, Tomino Y, Fogo AB, et al. IgA nephropathy. Nat Rev Dis Primers. 2016;2:16001.

4. Trimarchi H, Barratt J, Cattran DC, Cook HT, Coppo R, Haas M, et al. Oxford classification of IgA nephropathy 2016: an update from the IgA nephropathy classification working group. Kidney Int. 2017;91(5):1014-21.

5. Coppo R, Troyanov S, Bellur S, Cattran D, Cook HT, Feehally J, et al. Validation of the Oxford classification of IgA nephropathy in cohorts with different presentations and treatments. Kidney Int. 2014;86(4):828-36.

6. Alamartine E, Sauron C, Laurent B, Sury A, Seffert A, Mariat C. The use of the Oxford classification of IgA nephropathy to predict renal survival. Clin J Am Soc Nephrol. 2011;6(10):2384-8.

7. Tanaka S, Ninomiya T, Katafuchi R, Masutani K, Tsuchimoto A, Noguchi H, et al. Development and validation of a prediction rule using the Oxford classification in IgA nephropathy. Clin J Am Soc Nephrol. 2013;8(12):2082-90.

8. Zhu X, Li H, Liu Y, You J, Qu Z, Yuan S, et al. Tubular atrophy/ interstitial fibrosis scores of Oxford classification combinded with proteinuria level at biopsy provides earlier risk prediction in $\lg \mathrm{A}$ nephropathy. Sci Rep. 2017;7(1):1100.

9. Iwano M, Neilson EG. Mechanisms of tubulointerstitial fibrosis. Curr Opin Nephrol Hypertens. 2004;13(3):279-84.

10. Harris RC, Neilson EG. Toward a unified theory of renal progression. Annu Rev Med. 2006;57:365-80.

11. Sörensen I, Susnik N, Inhester T, Degen J, Melk A, Haller H, et al. Fibrinogen, acting as a mitogen for tubulointerstitial fibroblasts, promotes renal fibrosis. Kidney Int. 2011;80(10):1035-44.

12. Huang MJ, Wei RB, Wang Y, Su TY, Di P, Li QP, et al. Blood coagulation system in patients with chronic kidney disease: a prospective observational study. BMJ Open. 2017;7(5):e014294.

13. Grandaliano G, Monno R, Ranieri E, Gesualdo L, Schena F, Martino C, et al. Regenerative and proinflammatory effects of thrombin on human proximal tubular cells. J Am Soc Nephrol. 2000;11(6):1016-25.

14. Ma Y, Zuo L, Chen J, Luo Q, Yu X, Li Y, et al. Modified glomerular filtration rate estimating equation for Chinese patients with chronic kidney disease J Am Soc Nephrol. 2006;17(10):2937-44.

15. Tanaka K, Sugiyama H, Yamanari T, Mise K, Morinaga H, Kitagawa M, et al. Rena expression of trefoil factor 3 mRNA in association with tubulointerstitial fibrosis in IgA nephropathy. Nephrology (Carlton, Vic). 2018;23(9):855-62.

16. Lee J, An J, Hwang J, Lee H, Lee J, Kim S. p38 MAPK activity is associated with the histological degree of interstitial fibrosis in IgA nephropathy patients. PLoS One. 2019;14(3):e0213981.

17. Wu C, Wang AY, Li G, Wang L. Association of high body mass index with development of interstitial fibrosis in patients with IgA nephropathy. BMC Nephrol. 2018;19(1):381.

18. Zhang J, Ren P, Wang Y, Feng S, Wang C, Shen $X$, et al. Serum matrix Metalloproteinase-7 level is associated with fibrosis and renal survival in patients with IgA nephropathy. Kidney Blood Press Res. 2017;42(3):541-52.

19. Pant A, Kopec AK, Luyendyk JP. Role of the blood coagulation cascade in hepatic fibrosis. Am J Physiol Gastrointest Liver Physiol. 2018;315(2):G171-G6.

20. Zheng Z, Ma T, Lian X, Gao J, Wang W, Weng W, et al. Clopidogrel reduces Fibronectin accumulation and improves diabetes-induced renal fibrosis. Int J Biol Sci. 2019;15(1):239-52.

21. Favreau F, Thuillier R, Cau J, Milin S, Manguy E, Mauco G, et al. Anti-thrombin therapy during warm ischemia and cold preservation prevents chronic kidney graft fibrosis in a DCD model. Am J Transplant. 2010;10(1):30-9.

22. Northup PG. Hypercoagulation in liver disease. Clin Liver Dis. 2009;13(1):109-16.

23. Suarez-Alvarez B, Liapis $\mathrm{H}$, Anders HJ. Links between coagulation, inflammation, regeneration, and fibrosis in kidney pathology. Lab Investig. 2016;96(4):378-90.

24. Sultan P, Butwick A. Platelet counts and coagulation tests prior to neuraxial anesthesia in patients with preeclampsia: a retrospective analysis. Clin Appl Thromb Hemost. 2013;19(5):529-34.

25. Liu N, Ono T, Suyama K, Nogaki F, Shirakawa K, Maeda M, et al. Mesangial factor $\vee$ expression colocalized with fibrin deposition in IgA nephropathy. Kidney Int. 2000;58(2):598-606.

26. Ono T, Liu N, Kasuno K, Kusano H, Nogaki F, Kamata T, et al. Coagulation process proceeds on cultured human mesangial cells via expression of factor V. Kidney Int. 2001;60(3):1009-17.

27. Madhusudhan T, Kerlin BA, Isermann B. The emerging role of coagulation proteases in kidney disease. Nat Rev Nephrol. 2016;12(2):94-109.

28. Tsushima K, Yamaguchi K, Kono Y, Yokoyama T, Kubo K, Matsumura T, et al. Thrombomodulin for acute exacerbations of idiopathic pulmonary fibrosis: a proof of concept study. Pulm Pharmacol Ther. 2014;29(2):233-40.

29. Fujii M, Hayakawa H, Urano T, Sato A, Chida K, Nakamura H, et al. Relevance of tissue factor and tissue factor pathway inhibitor for hypercoagulable state in the lungs of patients with idiopathic pulmonary fibrosis. Thromb Res. 2000;99(2):111-7.

30. Tripodi A, Anstee QM, Sogaard KK, Primignani M, Valla DC. Hypercoagulability in cirrhosis: causes and consequences. J Thromb Haemost. 2011;9(9):1713-23.

31. Wang $H$, Zheng $C$, Lu Y, Jiang $Q$, Yin $R$, Zhu $P$, et al. Urinary fibrinogen as a predictor of progression of CKD. Clin J Am Soc Nephrol. 2017;12(12):1922-9.

32. Li S, Gao Y, Shao M, Tang B, Cao W, Sun X. Association between coagulation function and patients with primary angle closure glaucoma: a 5-year retrospective case-control study. BMJ Open. 2017;7(11):e016719.

33. Liu N, Mori N, lehara N, Uemura K, Fukastu A, Kita T, et al. Soluble fibrin formation in the mesangial area of IgA nephropathy. Clin Exp Nephrol. 2007;11(1):71-6. 
34. Yin J, Wang F, Kong Y, Wu R, Zhang G, Wang N, et al. Antithrombin II prevents progression of chronic kidney disease following experimental ischaemic-reperfusion injury. J Cell Mol Med. 2017;21 (12):3506-14.

35. Dong J, Duan X, Feng R, Zhao Z, Feng X, Lu Q, et al. Diagnostic implication of fibrin degradation products and D-dimer in aortic dissection. Sci Rep. 2017;7:43957.

36. Tan X, Chen G, Liu Y, Zhou L, He L, Liu D, et al. Serum D-dimer is a potential predictor for thromboembolism complications in patients with renal biopsy. Sci Rep. 2017;7(1):4836

37. Li P, Ho K, Szeto C, Yu L, Lai F. Prognostic indicators of IgA nephropathy in the Chinese--clinical and pathological perspectives. Nephrol Dial Transplant. 2002;17(1):64-9.

38. Liu D, You J, Liu Y, Tang X, Tan X, Xia M, et al. Serum immunoglobulin G provides early risk prediction in immunoglobulin a nephropathy. Int Immunopharmacol. 2019;66:13-8.

39. Liu $Y$, Zhang $Y$, Liu D, Tan $X$, Tang $X$, Zhang F, et al. Prediction of ESRD in IgA nephropathy patients from an Asian cohort: a random Forest model. Kidney Blood Press Res. 2018;43(6):1852-64.

40. Huang M, Wei R, Wang Z, Xing Y, Gao Y, Li M, et al. Mechanisms of hypercoagulability in nephrotic syndrome associated with membranous nephropathy as assessed by thromboelastography. Thromb Res. 2015;136(3): 663-8.

41. Mahmoodi BK, ten Kate MK, Waanders F, Veeger NJGM, Brouwer J-LP, Vogt $L$, et al. High absolute risks and predictors of venous and arterial thromboembolic events in patients with nephrotic syndrome: results from a large retrospective cohort study. Circulation. 2008:117(2):224-30.

42. Kerlin BA, Waller AP, Sharma R, Chanley MA, Nieman MT, Smoyer WE. Disease severity correlates with thrombotic capacity in experimental Nephrotic syndrome. J Am Soc Nephrol. 2015;26(12):3009-19.

43. Kerlin B, Ayoob R, Smoyer W. Epidemiology and pathophysiology of nephrotic syndrome-associated thromboembolic disease. Clin J Am Soc Nephrol. 2012;7(3):513-20.

44. Lutz J, Menke J, Sollinger D, Schinzel H, Thürmel K. Haemostasis in chronic kidney disease. Nephrol Dial Transplant. 2014;29(1):29-40.

45. Weber J, Olyaei A, Shatzel J. The efficacy and safety of direct oral anticoagulants in patients with chronic renal insufficiency: a review of the literature. Eur J Haematol. 2019:102(4):312-8.

46. Yoshikawa N, Ito H, Sakai T, Takekoshi Y, Honda M, Awazu M, et al. A controlled trial of combined therapy for newly diagnosed severe childhood IgA nephropathy. The Japanese pediatric IgA nephropathy treatment study group. J Am Soc Nephrol. 1999;10(1):101-9.

47. Shima Y, Nakanishi K, Kaku Y, Ishikura K, Hataya H, Matsuyama T, et al. Combination therapy with or without warfarin and dipyridamole for severe childhood IgA nephropathy: an RCT. Pediatr Nephrol. 2018;33(11):2103-12.

48. Zhai Y, Zhu L, Shi S, Liu L, Lv J, Zhang H. Elevated soluble VEGF receptor sFlt-1 correlates with endothelial injury in IgA nephropathy. PLoS One. 2014; 9(7):e101779.

49. Kusano T, Takano H, Kang D, Nagahama K, Aoki M, Morita M, et al. Endothelial cell injury in acute and chronic glomerular lesions in patients with IgA nephropathy. Hum Pathol. 2016:49:135-44.

50. Liu D, Liu Y, Chen G, He L, Tang C, Wang C, et al. Rapamycin enhances repressed autophagy and attenuates aggressive progression in a rat model of IgA nephropathy. Am J Nephrol. 2017;45(4):293-300.

51. Wu H, Wang $Y$, Zhang $Y$, Xu F, Chen J, Duan $L$, et al. Breaking the vicious loop between inflammation, oxidative stress and coagulation, a novel antithrombus insight of nattokinase by inhibiting LPS-induced inflammation and oxidative stress. Redox Biol. 2020;32:101500.

52. Chen G, Chen H, Wang C, Peng Y, Sun L, Liu H, et al. Rapamycin ameliorates kidney fibrosis by inhibiting the activation of mTOR signaling in interstitial macrophages and myofibroblasts. PLoS One. 2012;7(3):e33626.

\section{Publisher's Note}

Springer Nature remains neutral with regard to jurisdictional claims in published maps and institutional affiliations.

\section{Ready to submit your research? Choose BMC and benefit from:}

- fast, convenient online submission

- thorough peer review by experienced researchers in your field

- rapid publication on acceptance

- support for research data, including large and complex data types

- gold Open Access which fosters wider collaboration and increased citations

- maximum visibility for your research: over $100 \mathrm{M}$ website views per year

At $\mathrm{BMC}$, research is always in progress.

Learn more biomedcentral.com/submissions 